\title{
Verpackung im Spannungsfeld zwischen Marketing und Produktion
}

\author{
Jürg Gutzwiller*
}

In einer 1989 in der Schweiz durchgeführten Meinungsumfrage, in der nach spontanen Assoziazionen zum Thema "Verpackung» gefragt wurde, nannten $56 \%$ der Befragten das Schlagwort «Vergeudung" und $15 \%$ den Begriff "Umweltbelastung». Obwohl im Teil mit'vorgegebenen Antworten die Begriffe "Produkteschutz» und "Warenverteilung» höher gewichtet wurden, zeigen die spontanen Äusserungen die stark emotionelle Komponente, die die Verpackung beim Verbraucher hervorruft. In der Tat ist der heutige Konsument täglich mit Verpackungen, die ihren Dienst geleistet haben und die somit zum Abfall geworden sind, konfrontiert. Deswegen jedoch ohne weiteres Nachdenken die Verpackung zu verteufeln zeugt von bedenklicher Oberflächlichkeit, stellt doch die Vielfalt des Verpackungsangebots ein wesentliches Stück unserer persönlichen Freiheit dar. Denn erst durch die Verpackung wird ein Produkt zur Ware. Beim Vorgang des Auspackens findet jedoch oft ein entscheidender Wandel statt: der Enthusiast wird nicht selten zum Gegner der Güter.

Vom breiten Publikum wird die Leistung der Verpackung meist vollständig ignoriert oder sie wird bestenfalls als notwendiges Übel abgetan. Auch der Chemiker denkt logischerweise in den Kategorien von Reagentien, Edukten, Zwischen- und Endprodukten und nimmt die zu deren Aufbewahrung notwendigen verschiedenen Gebinde als selbstverständlich hin. Erst wenn ein Präparat nach einiger Zeit sich zu zersetzen beginnt oder wenn die vom Lager angelieferten Deckel nicht richtig auf die zugehörigen Fässer passen, beginnt er sich mit der Verpackung auseinanderzusetzen.

In Wirklichkeit ist das Segment der Verpackung ein äusserst wichtiger Wirtschaftsfaktor, der in der Schweiz inklusive Abpackung mit $\mathrm{ca}$. 6 Milliarden Franken und $c a .30000-35000$ Beschäftigten nicht $\mathrm{zu}$ hoch angesetzt ist. Entsprechend sind die Aufgaben und Leistungen der Ver-

\footnotetext{
* Korrespondenz: Dr. J. Gutzwille

F. Hoffmann-La Roche $A G$

Postfach

$\mathrm{CH}-4002$ Basel
}

packung im Marketing-Mix anzusiedeln. Welch gewaltiger Strukturwandel ist in den vergangenen Jahrzehnten erfolgt, von der Versorgung der Bevölkerung innerhalb der Kirchturm-Region mit weitgehender regionaler Autarkie bis zur weltweiten Warenverteilung. Ihre Logistik wäre ohne die heutige Vielfalt des Verpackungsangebots undenkbar. Man denke nur an unsere reibungslos funktionierenden Selbstbedienungsläden, an den Nutzen einer sterilen Injektion an einer abgelegenen Unfallstelle oder an die Selbstverständlichkeit, dass ein aus Japan importiertes Stereogerät beim ersten Einschalten funktioniert. Auch ist die Verpackung keineswegs Materialverschleiss oder Kostenverursacher. Im FoodBereich beispielsweise werden durch die industrielle Verarbeitung und Verpackung im Gegenteil jährlich riesige Tonnagen hochwertiger Lebensmittel vor Verderb geschützt. Auch ist die industrielle Abpakkung Voraussetzung für eine rationelle, kostengünstige Distribution von Massenprodukten. Die Tatsache, dass einzelne Luxusgüter in Luxusverpackungen vermarktet werden, ist nur die berühmte Ausnahme, die die Regel bestätigt.

Der Lebenszyklus einer Verpackung besteht nicht nur aus Entnahme aus dem Verkaufsregal, Mitnahme nach Hause, Auspacken und Wegwerfen des Packmaterials. $\mathrm{Er}$ ist vielmehr eine Kette festgefügter Glieder, von der Packstoff- über die Packmittelherstellung, das Abpacken, die Verteilung, den Verkauf, die Entnahme des Füllgutes bis zur Entsorgung des zum Abfall gewordenen Packmaterials. Idealerweise heisst Entsorgung heute Wiederverwertung, entweder dank des Einsatzes von Mehrwegsystemen oder aber durch stoffliche oder energetische Rezyklierung. Dabei ist es absolut unabdingbar, dass die einzelnen Glieder fein aufeinander abgestimmt sind. Was würde denn ein Packstoff taugen, der an das Füllgut unzulässige Mengen eines Additives abgibt, oder eine Verpackung, die das Produkt während der Dauer der Laufzeit nicht vor schädlichen Einflüssen schützt?

Die Verpackung als integrierter Bestandteil einer Handelsware muss eine ganze Reihe von Funktionen erfüllen. Dazu gehören Schutz des Produktes, Schaffen

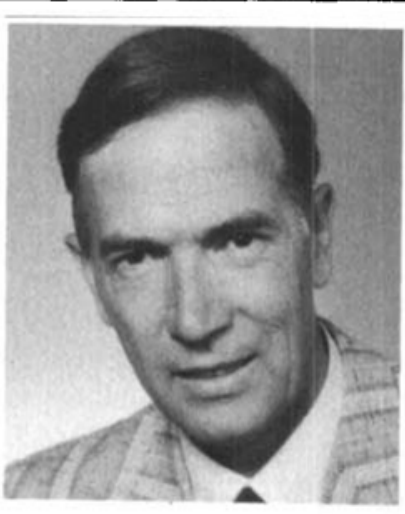

Jürg Gutzwiller: Geboren 1937. Schulen und Chemiestudium in Basel. Nach der Promotion, 1964 wissenschaftlicher Mitarbeiter an der Universität Basel und Postdoctoral Fellow an der Stanford University, USA. 1966 Eintritt in die chemische Forschung der F. Hoffmann-La Roche AG in Nutley, USA. 1971 Wechsel innerhalb der Roche-Forschung nach Basel. Seit 1976 im technischen Management, zuerst Kontrollanalytik, seit 1983 Leiter der Abteilung Verpackungstechnik innerhalb der Pharmaproduktion. Zurzeit Präsident des Schweizerischen Verpackungsinstituts (SVI), der Interessengemeinschaft des schweizerischen Verpackungswesens.

der Voraussetzung für eine rationelle Logistik, Minimierung der Gesamtkosten, optimale Entsorgbarkeit sowie die für den Markterfolg vieler Verbrauchsgüter ausschlaggebenden Funktionen der Kommunikation und der Convenience für den Verbraucher. Entsprechend vielfältig und divergierend sind aber auch die äusseren Einflüsse, die bei der Gestaltung einer Pakkung zu berücksichtigen sind: so zum Beispiel behördliche Vorschriften bezüglich Kompatibilität von Lebensmitteln und Pharmazeutika mit dem Primärpackmittel, bezüglich Tauglichkeit von Transportverpackung gefährlicher Güter, bezüglich Deklaration und Kennzeichnung von Verkaufspackungen, aber auch Vorgaben der Abfallwirtschaft für die Entsorgung sowie die Bedürfnisse des Handels und die Wünsche des Verbrauchers. In der Organisation des Produzenten vertritt das Marketing vorwiegend die Belange des Verbrauchers, denn jedes Produkt muss - je nach dessen Art mehr oder weniger - mit der Verpackung um die Gunst des Konsumenten werben. Die übrigen Belange, meistens auch die Minimierung des Anteils der Verpackung an den Gesamtkosten, müssen von der Produktionsabteilung wahrgenommen werden. Weil nun aber die Interessen des Marketing primär auf die Profilierung des einzelnen Produktes im Sinne von Customer Focus, diejenigen der Produktion jedoch auf Standardisierung der Packungen und der Verpackungsabläufe ausgerichtet sind, bestehen innerhalb der produzierenden Firma oft echte Interessenkonflikte.

Anhand dieser Verpackungsfunktionen sollen nun doch einige Problemkreise der Verpackung pharmazeutischer Fertigspezialitäten angeschnitten werden. Zuerst zur Schutzfunktion vor Feuchtigkeit, Sauerstoff sowie partikulärer und mikrobiologi- 
scher Kontamination, die durch die Wahl der geeigneten Primärpackmittel gewährleistet wird. Für diese kommen die Packstoffe Glas, Kunststoffe, Elastomere, Aluminium sowie zu einem geringen Anteil $\mathrm{Pa}$ pier zum Einsatz. Dazu nur eine Bemerkung zu den für flexible Packungen verwendeten Folienmaterialien, hat doch die in Europa zurzeit sehr aktuelle Kontroverse um das PVC inzwischen auch die für feste orale Darreichungsformen wichtigste Verpackungsart, die Durchdrückpackung, erfasst. Da die halogenierten Polyolefine als einzige Polymere jedoch genügend hohe Sperreigenschaften für Wasserdampf aufweisen, würde deren generelles Verbot die von Spitälern und Patienten aus verschiedenen Gründen weit bevorzugte Blisterpackung praktisch verschwinden lassen. PVC als Trägerfolie liesse sich zwar noch mit dem entsprechenden Aufwand durch Polypropylen oder Polyester ersetzen, die Sperreigenschaften dieser Materialien wären aber ohne Beschichtung mit Polyvinylidenchlorid nicht für alle Produkte ausreichend. Die Folge wäre, dass viele empfindliche Präparate nur noch im bewährten Tablettenglas oder aber in einer flexiblen Packung angeboten werden könnten, die beidseitig Aluminium enthält. Aufgrund ihrer ausgezeichneten Materialeigenschaften sind also halogenierte Kunststoffe sowie aus verschiedenen Polymeren zusammengesetzte Verbundfolien für die flexiblen Verpackungen im Pharma- sowie im Food-Bereich absolut unentbehrlich.

Die Funktion der Minimierung der Gesamtkosten durch rationelle Abpacktechnik und Standardisierung der Packmittel stellt die Konzernzentrale eines multinationalen Pharmaunternehmens vor ganz spezielle Probleme. Bedingt durch die weltweite Dezentralisation der pharamzeutischen Produktion bleiben dem Stammhaus nur die Versorgung der kleinen Märkte sowie die Herstellung kleiner Produkte für Märkte der produzierenden Filialen. Entsprechend ungünstig präsentiert sich die Struktur der Verpackungsaufträge. Bei Roche werden in Basel auf 26 Verpakkungslinien jährlich ca. 75 Millionen Pakkungen hergestellt, die sich aus 32 verschiedenen Arten von Verpackungen mit 640 verschiedenen Packungsgrössen und insgesamt 2900 verschiedenen sprachlichen Aufmachungsvarianten sowie ca. 5200 verschiedenen bedruckten und unbedruckten Packmitteln zusammensetzen. Spannun- gen zwischen Produktion und Marketing ergeben sich zwangsweise im Zusammenhang mit Wünschen nach neuen, nicht standardmässigen Verpackungen, bei Fragen der Sortimentsvielfalt sowie wegen der unterschiedlichen Qualität der Bedarfsprognosen. Patentrezepte zur Verkürzung der Durchlaufzeiten, zur Reduktion der Lagerhaltung, d. h. zur Einführung von Justin-Time-Konzepten sowie zur Standardisierung der Packungen und damit der Verpackungsabläufe gibt es keine. Nur ausgeklügelte, den einzelnen Problemkreisen angepasste Teillösungen für die verschiedenen Stufen der hausinternen Logistik resp. Abstriche an den produktspezifisch ausgelegten Packungen können die gesamte Effizienz verbessern.

Der Ökofunktion der Verpackung wird heute auch in der Pharmaindustrie grösste Beachtung geschenkt. In diesem Bereich haben die schweizerischen LebensmittelGrossverteiler in den letzten Jahren bereits Wesentliches geleistet. Die Möglichkeiten sind in allen Industriezweigen die gleichen: Minimierung der Wandstärken von Hohlkörpern, nur soviel Produkteschutz, d.h. Packmittel mit hohen und höchsten Barriereeigenschaften, wie nötig, teilweiser Ersatz von Kunststoff durch Karton bei Sekundärpackungen, z. B. bei Ampullenpakkungen, und konsequente Berücksichtigung der Resultate von Ökobilanzen. Die Anwendung dieser Prinzipien ergibt nur soviel Verpackung wie nötig und bringt häufig auch gleichzeitig die kostengünstigste Verpackungslösung. Wegen ungenügender Dichte der Verpackungsabfälle von Pharmapräparaten der einzelnen Hersteller ist im Pharmabereich jedoch an den Einsatz von Mehrweggebinden nicht zu denken.

In der Convenience-Funktion der Pharmaverpackung wurde in den letzten Jahren bei Hoffmann-La Roche in Zusammenarbeit zwischen Marketing und Produktion unter Berücksichtigung der anderen Funktionen viel getan. Dabei sind z. B. zu erwähnen die konsequente Einführung von Garantieverschlüssen für alle Flaschen, Tuben und Hülsen, die Blisterpackungen mit Einzelperforation und Beschriftung der Einzelhöfe, welche für Spitäler Unitdose-Funktion haben und es auch dem ambulanten Patienten erlauben, den Inhalt geeignet zu portionieren, sowie die Umstellung auf Tubenpackungen mit leicht handhabbaren Stehverschlüssen oder die Rheu- matiker-gerechten Packungen der verschiedenen galenischen Darreichungsformen des neuen Entzündungshemmers Tilcotil.

Die Funktion der Kommunikation hat für pharmazeutische Präparate einen ganz anderen Stellenwert als für normale Konsumgüter. Jegliche Art von Information, die als Werbung aufgefasst werden könnte, ist selbstverständlich strikte verboten. Trotzdem kann die Kommunikation in und auf Pharmapackungen besser oder weniger gut sein. Bei Roche wurden in letzter Zeit manche Verbesserungen eingeführt, so z. B. die freiwillige Einführung der offenen Verfalldaten auf allen Packungen, die von der IKS mittlerweile vorgeschriebenen patientenfreundlichen Packungsprospekte mit für den Laien verständlichen Texten, oder die Abgabe von Zusatzetiketten bei Ampullenpackungen, die für den Operationssaal bestimmt sind, mit denen die injektionsbereiten Spritzen gekennzeichnet werden können. Weitere Massnahmen wurden von der IKS resp. vom Handel vorgeschrieben, so die Deklaration der potentiell allergenen Hilfsstoffe resp. der beim Apotheker elektronisch lesbare EAN-Strichcode. Auch bei der graphischen Packungsgestaltung wird laufend nach Verbesserungen der Produktekennzeichnung gesucht, insbesondere bei Präparaten mit verschiedenen Dosierungen.

Die Anforderungen, die von den verschiedenen Seiten an eine Verpackung gestellt werden, gehen oft diametral auseinander. Solange sie vorwiegend technischer Natur sind, lassen sie sich meist auf einen Nenner bringen. Schwieriger wird es, wenn die Forderungen ins Politische und Emotionelle abgleiten, wie dies bei der leidigen PVC-Diskussion der Fall ist. Neue Verpackungslösungen stossen aber rasch auch an die Grenzen des kostenmässig machbaren, dies besonders für abpackende Betriebe, die einen komplexen Produktemix mit einem entsprechend vielfältigen $\mathrm{Ma}$ schinenpark aufweisen. Wenn dazu für das neue, speziell zu verpackende Produkt nur mässige Verkaufsschätzungen vorliegen, so ist der Spielraum für innovative Lösungen oft so eng, dass bestenfalls Kompromisse resultieren, die sich stark an das Bestehende anlehnen. Die Verpackung reduziert sich im Spannungsfeld zwischen Marketing und Produktion eben sehr oft von ideenmässigen Höhenflügen auf die realistische Kunst des Machbaren. 